\title{
PRIMARY CENTRAL NERVOUS SYSTEM LYMPHOMA IN AN IMMUNOCOMPETENT HOST
}

\author{
Felipe Slaviero', Leonardo Frighetto², Jairo Júnior Casali', \\ Moema Nenê Santos ${ }^{3}$, José Ricardo Vanzin², Nério D. Azambuja Jr ${ }^{2}$
}

Primary central nervous system lymphoma (PCNSL) is a form of extranodal non-Hodgkin's lymphoma, which occurs in the brain, leptomeninges, spinal cord, or eyes. PCNSL is an uncommon intracranial neoplasm, particularly in the immunocompetent patient population. An immunosuppressive state is the unique established risk factor for PCNSL, and the HIV-related primary lymphoma is 3600 -fold more common compared with the general population. This tumor constitutes $0.3-1.5 \%$ of all intracranial neoplasm in patients without AIDS'. The age-adjusted incidence in the United States has augmented 3 folds in the last three decades, passing from 0.16 per 100000 between 1973-1984 to 0.48 per 100000 between $1985-1997^{2}$, although current data suggests a plateau or a slightly decrease in the annual incidence.

The purpose of this study is to report and discuss the uncommon occurrence of PCNSL in an immunocompetent patient.

\section{CASE}

The patient agreed with the publication of this study under informed consent.
A 31-year-old caucasian woman, with no previous medical history, presented with a 12 months history of personality changes, cognitive dysfunction and paroxysmal impairment of consciousness. Constitutional symptoms defined as "B" symptoms (loss of $10 \%$ of body weight and drenching night sweats) were present, but no fever. Neurological examination showed cognitive dysfunction, gait ataxia and absence of signs indicating intracranial hypertension. There was no peripheral adenopathy or hepatosplenomegaly.

Computed tomography (CT-scan) showed an expansive mass in the white matter of the left cerebral hemisphere (Fig 1A). T1weighted magnetic resonance imaging (MRI) after gadolinium contrast administration showed bilateral homogenous lesions (Fig 1B).

HIV and hepatitis sorology were negative. Histopathological evaluation after a stereotactic biopsy of the tumor disclosed diffuse pleomorphic cells with large nuclei and a coarse chromatin pattern (Fig 2A). The immunohistochemical reaction was characteristic of diffuse large B-cell lymphoma (Fig 2B).

Further investigation with CT-scan of the chest, abdomen and pelvis, as well as bone marrow biopsy, evaluation of cerebrospinal fluid (CSF) and ophthalmologic examination were normal.

An Ommaya reservoir was implanted for intraventricular chemotherapy with methotrexate (MTX) and dexamethasone.

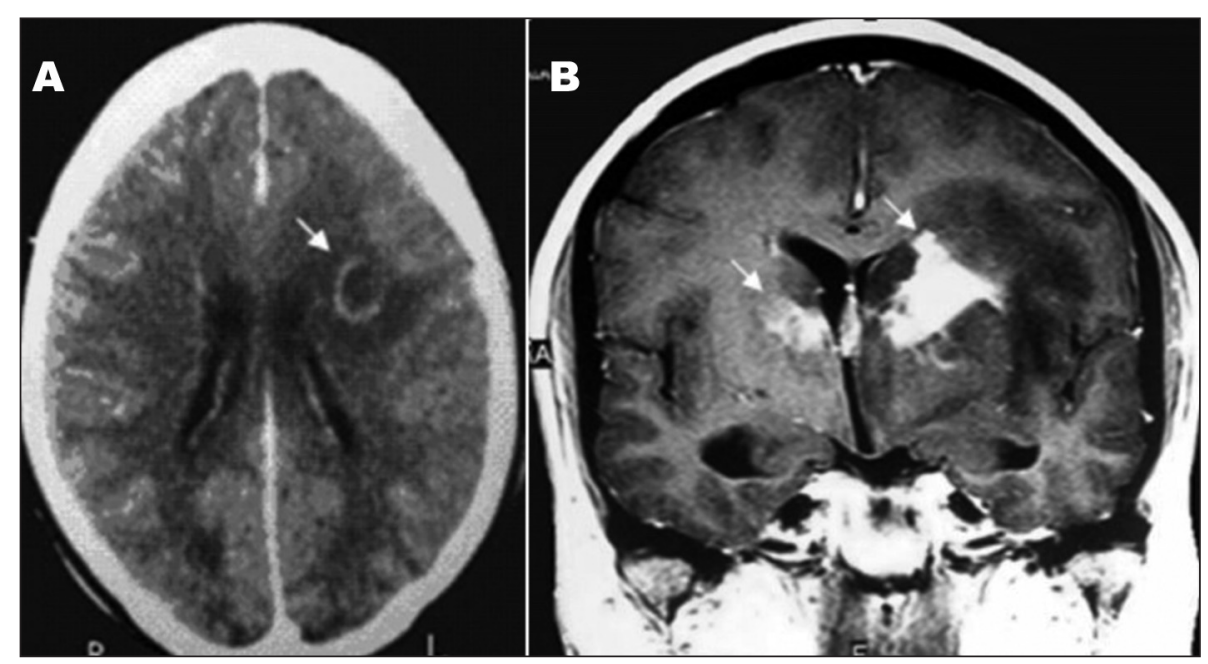

Fig 1. (A) CT-scan: expansive mass on the left cerebral hemisphere (arrow). (B) T7-weighted MRI: bilateral homogeneously-enhanced lesions (arrows).

\section{LINFOMA PRIMÁRIO DO SISTEMA NERVOSO CENTRAL EM PACIENTE IMUNOCOMPETENTE}

Neurology and Neurosurgery Service, Hospital São Vicente de Paulo, Passo Fundo RS, Brazil: 'Medical Student, Universidade de Passo Fundo, Passo Fundo RS, Brazil; ${ }^{2}$ Neurosurgeon, Neurology and Neurosurgery Service, Hospital São Vicente de Paulo; ${ }^{3}$ Hematologist, Hospital São Vicente de Paulo.

Received 10 March 2008, received in final form 30 May 2008. Accepted 18 June 2008.

Dr. Felipe Slaviero - Edifício das Clínicas - Rua Teixeira Soares 885 / 10 9 andar - 99010-901 Passo Fundo RS - Brasil. E-mail: felipeslavier@yahoo.com.br 


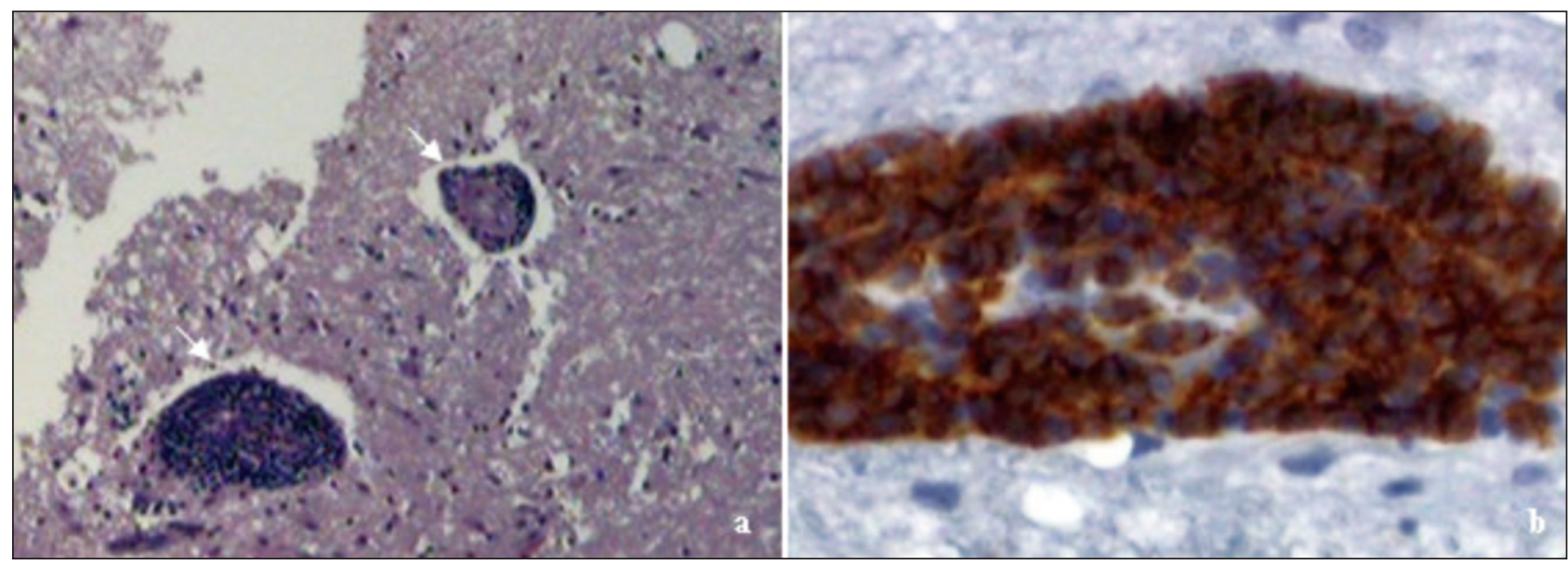

Fig 2. (A) Histopathological analysis: lymphoid clustering around the small cerebral vessels (arrows) H\&E 100x; (B) Immunohistochemical study: lymphocytes with ovoid nucleus, scarce cytoplasm and CD 20 positive reaction, 400x.

Adjuvant systemic chemotherapy was performed using highdose MTX, vincristine, procarbazine and cytarabine. The patient was submitted to fractionated whole-brain radiotherapy with 36 Gy. An eight months follow-up CT-scan showed disease control without corticosteroid therapy dependence. There was no evidence of neurotoxicity.

\section{DISCUSSION}

PCNSL represents $2.7 \%$ of all primary brain tumors. In $90 \%$ it is a diffuse large B-cell lymphoma. The remaining are poorly characterized low-grade lymphomas, Burkitt's lymphoma, and T-cell lymphomas ${ }^{3}$. In immunocompetent hosts, the median age at diagnosis is 53 to 57 years, with a slight male predominance ${ }^{4}$. The classical clinical presentation relates to the location in the central nervous system (CNS), without "B symptoms" (weight loss, night sweats and fever). Bataille et al. ${ }^{5}$, in a study with immunocompetent patients with $P C N S L$, reported that $70 \%$ had focal neurologic deficits, $43 \%$ had neuropsychiatric symptoms, $33 \%$ had increased intracranial pressure, $14 \%$ had seizures, and $4 \%$ had ocular symptoms.

Laboratory evaluation should include lactate dehydrogenase, determination of hepatic, renal and thyroid function, HIV sorology, electrolytes analysis and reactive $\mathrm{C}$ protein. To determine the disease extension, a detailed ophthalmologic evaluation, CSF analysis, bone marrow biopsy, CT-scan of the chest, abdomen, and pelvis, and brain MRI are recommended. Occult extraneural disease in this screening is found in 3.9 to $12.5 \%$ of the patients ${ }^{6}$, and systemic dissemination is discovered in $10 \%$ during the course of the disease ${ }^{7}$.

If lumbar puncture can be performed safely, routine CSF analysis should be done. Pleocytosis, high protein concentrations and low glucose levels are often present, whereas the cytology typically shows clumped pleomor- phic cells with enlarged nuclei and coarse chromatin in $26 \%$ to $31 \%$ of PCNSL patients ${ }^{7}$.

The CT-scan may reveal isodense to hyperdense images. T1-weighted MRI demonstrates hypointense lesions while the T2-weighted MRI shows isointense to hyperintese images, secondary to the tumor's high cell density.

Kuker et al. ${ }^{8}$, in a MRI study reported solitary lesions in two thirds of the patients. Lesions were located in the cerebral hemispheres (38\%), thalamus and basal ganglia $(16 \%)$, corpus callosum (14\%), ventricular region (12\%), and cerebellum (9\%). Contrast enhancement was strong in $85 \%$ and absent in only $1 \%$ of the patients.

Stereotactic needle biopsy is the standard procedure to provide tumor specimen for histopathologic diagnosis. The role of the surgical resection alone is limited, with a median survival time of less than four months.

The lymphomas are one of the most sensitive primary CNS tumors to a large variety of treatments. These include corticosteroids, radiation therapy, chemotherapy and, combined-modality therapy.

Dexamethasone is associated with an initial response rate of $70 \%$, usually transient. Corticosteroids should be avoided before the stereotactic procedure because it may interfere with the diagnosis.

Radiation therapy provides complete radiographic and clinical response ranging from $60 \%$ to $90 \%$ of patients treated with standard fractionation. Despite this initial response, about $90 \%$ recur within one year, with a median survival time of 10 to 18 months?.

MTX is currently the mainstay of the treatment, but has a poor penetration through the blood-brain barrier, justifying the intraventricular chemotherapy in addition to the intravenous administration. Complete radiographic 
response with chemotherapy alone ranges from $30 \%$ to $100 \%$ of the patients ${ }^{10}$.

Combined-modality therapy (chemotherapy plus radiation therapy) has achieved high response rates, improving the survival to 60 months. Because of the potential risk of severe late toxicity, which is age-related, this treatment is more indicated in young patients ${ }^{10}$. Multiagent MTX-based chemotherapy is currently the treatment of choice ${ }^{6}$. Ancillary treatment with other chemotherapeutic agents can be used, but decisions need to be made on a case-by-case basis and should be reserved to young patients without poor prognosis risk factors, as the presenting one.

Neurotoxicity manifests as ataxia, cognitive impairment, and incontinence. This devastating complication occurs in up to $30 \%$ of the patients and often happens in patients older than 60 years, in a mean period of 13.2 months after the treatment ${ }^{10}$.

Although currently available therapeutic regimens prolong survival, they are not curative. A complete response is defined as no brain imaging enhancing disease, corticosteroid independence, normal ophthalmologic examination, and negative CSF cytology. These purposes were successfully accomplished in this case.
ACKNOWLEDGEMENT - We acknowledge Senair Alberto Ambros, MD for the neuroimaging descriptions and Elder Lersch, MD for the providing of histopathological images.

\section{REFERENCES}

1. Sonstein W, Tabaddor K, Llena JF. Solitary primary CNS lymphoma: long term survival following total resection. Med Oncol 1998; 15:61-65.

2. Olson JE, Janney CA, Rao RD, et al. The continuing increase in the incidence of primary central nervous system non-Hodgkin lymphoma. Cancer 2002; 95:1504-1510.

3. Miller DC, Hochberg FH, Harris NI, et al. Pathology with clinical correlations of primary central nervous system non-Hodgkin's lymphoma: the Massachucetts General Hospital experience 1958-1989. Cancer 1994; 74:1383-1397.

4. Schabet M. Epidemiology of primary CNS lymphoma. J Neurooncol 1999; 43:199-201.

5. Bataille B, Delwail V, Menet E, et al. Primary intracerebral malignant lymphoma: a report of 248 cases. J Neurosurg 2000; 92:261-266.

6. Batchelor T, Loeffler JS. Primary CNS lymphoma. J Clin Oncol 2006; 24:1281-1288.

7. Fitzsimmons A, Upchurch K, Batchelor TT. Clinical features and diagnosis of primary central nervous system lymphoma. Hematol Oncol Clin N Am 2005;19:689-703.

8. Kuker W, Nagele T, Korfel A, et al. Primary central nervous system lymphomas (PCNSL): MRI features at presentation in 100 patients. J Neurooncol 2005;72:169-177.

9. Nelson DF, Martz KL, Bonner H, et al. Non-Hodgkin's lymphoma of the brain: Can high dose large volume radiation therapy improve survival? Report on a prospective trial by the Radiation Therapy Oncology Group (RTOG): RTOG 8315. Int J Radiat Oncol Biol Phys 1992; 23:9-17.

10. Ferreri AJM, Abrey LE, Blay J-Y, et al. Management of primary central nervous system lymphoma: a summary statement from the $8^{\text {th }}$ International Conference on Malignant Lymphoma. J Clin Oncol 2003; 21:2407-2414. 